1

\title{
Low cost high intensity LED illumination device for high uniformity laboratory purposes
}

\author{
Carmine D'Alessandro ${ }^{1,}{ }^{*}$, Davide De Maio ${ }^{1,2}$, Teresa Mundo $^{3}$, Marilena Musto ${ }^{1}$, Francesco Di \\ Giamberardino $^{3}$, Matteo Monti ${ }^{3}$, Davide Dalena ${ }^{3}$, Vittorio G Palmieri ${ }^{3}$, Daniela De Luca ${ }^{4,2}$, \\ Emiliano Di Gennaro ${ }^{4}$ and Roberto Russo ${ }^{2}$ \\ 1 Industrial Engineering Department, University of Napoli “Federico II", Napoli, Italy \\ 2 Istituto di Scienze Applicate e Sistemi Intelligenti, Unità di Napoli \\ 3 TVP Solar SA, 10 rue du Pré-de-la-Fontaine ZIMEYSA 1242 Satigny (GE), Switzerland \\ 4 Physics Department, University of Napoli “Federico II", Napoli, Italy \\ *Correspondence: carmine.dalessandro2@unina.it
}

\begin{abstract}
Uniform illumination is a key requirement in different research fields. However, this requirement is often difficult to achieve when high intensity is required at the same time. Recent advancements in LED lamps allow nowadays for compact and economical solutions. In this work we present a suitable solution for various laboratory purposes requiring stable, uniform and high intensity illumination. The system is composed of four identical high power white LED arrays of $30 \mathrm{~mm}$ diameter each, placed on a supporting and cooling structure having a minimum volume of $26 \mathrm{~cm} \times 26 \mathrm{~cm} \times 8 \mathrm{~cm}$. A numerical model has been developed, based on a ray tracing software, in order to simulate the performances. These have then been experimentally validated with measurements of the power density map, carried out with a $1 \%$ uncertainty pyranometer. Data show that the built system is very stable over time and provides an illumination uniformity higher than $98 \%$, on a surface of $50 \mathrm{~mm}$ radius, which reduces to $95 \%$ on a surface of $75 \mathrm{~mm}$ radius. The power density can be adjusted in the 390-1360 $\mathrm{W} \mathrm{m}^{-2}$ range, not affecting uniformity.
\end{abstract}

Keywords: high power illumination; compact device; solar thermal simulator; light uniformity; low cost LED system

\section{Introduction}

Uniform illumination is a key requirement in several civil and industrial applications as well as in different research fields. Typically, it is obtained by multiple artificial lamps with an appropriate geometrical configuration, which in turn strongly depends on the required illuminating power density, energy efficiency and footprint constraints. The Light Emitting Diodes (LED) have represented a turning point in almost all applications where the light quality and energy saving was an issue, such as office or road illumination, where the quality is strongly correlated to the human eye comfort [1] and where there is also a particular attention toward the energy consumption [2]. Moreover, LEDs have had a strong impact also in several laboratory activities such as photo-chemical reactions, where uniformity, time stability and tunable intensity are imperative features to characterize a photo-reactive process [3]. In order to achieve the desired control over the photoreaction, transparent microfluidic channels are adopted. This kind of microreactor needs to be illuminated by a temporally stable and uniform light, so that the size of the illuminated area determines the number of channels that could be involved [4]. Recently, small LEDs placed on the top of the microfluid channels have also been developed to reduce energy consumption [5] and the correlation between the light intensity and the chemical reaction has been studied [6], pointing out the important role of light uniformity for this kind of applications. Photodynamic inactivation of bacteria has also benefit of high power LED illumination $[7,8]$ and, in both cases, good uniformity 
was achieved by using lens placed on top of planar led array on a small area of few $\mathrm{cm}^{2}$.

Another research field that has profit of LEDs is the photo-lithography [9], where high intensity 5x5 UV-LED arrays was combined with collimating lens: a uniformity of about $90 \%$ was obtained on an area of few $\mathrm{cm}^{2}$ by putting the array in rotation [10] and a rotating substrate allows to obtain 3D figures. Also in the machine vision field [11], new LED based solutions have recently been developed to improve the contrast between 2D samples and the background [12], or the detection of tiny part [13]. LEDs and LED arrays have also been used in vein detection [14] and other biomedical applications [15].

For solar panel testing, both photovoltaic and thermal [16-19], high intensity is an additional requirement. The sample has to be illuminated with a controlled and uniform power density as high as the $1000 \mathrm{~W} \mathrm{~m}^{-2}$ as provided by the Sun. In such a way, the electric or thermal output of the sample can be directly compared to the light input for efficiency measurements. Classes of solar simulators are defined according to international standard [20], taking into account 3 parameters: nonuniformity, spectral match and temporal instability. For a Class A, solar simulator non-uniformity and temporal instability should be less the $2 \%$, (less than $5 \%$ for class B and less than $10 \%$ for class C) [20]. Moreover, in photovoltaic cells the conversion efficiency is spectrally dependent and different lamps are used to reproduce the Sun spectrum: metal halide lamps $[19,21]$, high pressure xenon discharge bulb [22] or LEDs [23]. A combination of different light sources, such as LEDs and halogen or quartz lamp, can be operated together to be cost effective [17]. Most effort in this field has been devoted to the development of solar simulator to test photovoltaic devices [20]. The use of several LEDs, with different spectral emissions, was aimed to match the Sun spectrum. Typically, such solution requires a source area much larger than the illuminated one and it results in limited area of illumination and uniformity. Lopez-Fraguas et al. have recently presented a class A solar simulator based on LEDs, but the uniform area was limited to $1 \mathrm{~cm}^{2}$ [24]. Also Al-Ahmad et al. [25] have developed a "large area" class A solar simulator (98\% uniformity); however, the class A was limited to an area of $20 \mathrm{~cm}^{2}$, when extending to an area of $32 \mathrm{~cm}^{2}$ the uniformity reduces to Class B (95\% uniformity).

However, when the device under test has a response with a small wavelength dependence, even unmatched sources can be effectively used. This is the case of solar thermal device whose light source, used as solar simulator, is usually arc lamp or metal halide lamp [26]. Recently, Moss et al. [16] have presented a solar simulator to test selective solar absorbers placed in a flat vacuum envelope without concentration. It is constituted of four quartz halogen bulbs inserted in a reflecting tube to increase uniformity and energy efficiency. The system presents a very good uniformity on a large area, however it is bulky, heavy and with a quite low energy efficiency. Moreover, the use of halogen quartz lamps produces a large fraction of IR radiation, outside the Sun spectrum, that is absorbed by the cover glass which is opaque above about $2.5 \mu \mathrm{m}$ and it is responsible of an unwanted glass heating [16]. For this kind of applications, the adoption of high intensity white LED array can be very advantageous in term of unwanted IR radiation and system dimensions.

The illumination uniformity in most of the reported applications have been optimized using the analytical approaches [27] and several optimization methods have been developed [28-30]. Moreno et al. [31] analyzed linear, circular and square arrangements of small LED arrays finding analytically the maximum distance between two adjacent sources, still providing high uniformity on the illuminated surface. Other studies propose the use of lenses to obtain the required uniformity, minimizing the number of LEDs [32]. However, when the reflections have to be taken in to account, as it is the case in [16], a ray optic approach has to be preferred.

The main objective of the work was to develop a cheap and compact class A solar thermal simulator on an area of at least $100 \mathrm{~cm}^{2}$ and a class B on an area of at least $200 \mathrm{~cm}^{2}$. The proposed solution consists of four high power white LED arrays arranged in compact layout to be broadly applicable as multi-purposes workbench laboratory facility. The main application of the system is the measurement of absorptance and emittance in selective solar absorbers [33-36]. As described in the work [37], the observed difference in solar absorptance between optical and calorimetric measurements was due to a not perfect calibration of the adopted illumination system. To achieve 
reliable absorptance values, the light intensity impinging on the surface must be uniform and well calibrated. To take into account the reflection of the supporting structure, the system optimization has been obtained by numerical simulations with a ray tracing software. The presented system is also easily scalable to illuminate much larger area increasing the number of LED arrays and it has been used as calibrated radiation source for measurement of solar collector thermal efficiency.

\section{Design of an artificial light source system}

LED is a very powerful artificial light source with high electric-to-light conversion efficiency and low production cost. One of the main drawbacks of artificial lights, including LEDs, is the nonuniformity of light power density on the illuminated surface. In fact, LEDs can be considered Lambertian emitters, thus the angular distribution of emitted light follows a cosine law with viewing angle $\theta$, eq. 1 .

$$
E(r, \theta)=E_{0}(r) \cos ^{m} \theta
$$

where $r$ is the distance from the source, $E_{0}(r)$ is the corresponding irradiance on the axis and $m$ is equal to 1 for Lambertian sources [38].

Uniformity can be defined by several dimensionless parameters and each field of application requires the appropriate one [1]. These parameters typically depend on $E_{\max }$ and $E_{\min }$ which are the maximum and the minimum of the irradiance $E=E(r, \varphi)$ calculated on the illuminated surface. The most used indicators for uniformity and non-uniformity, eqs. 2 and 3 respectively, are:

$$
\begin{gathered}
\xi=\frac{E_{\min }}{E_{\max }} \\
\Delta E=\frac{E_{\max }-E_{\min }}{E_{\text {mean }}} \\
\text { where } \quad E_{\text {mean }}(R)=\frac{\int_{0}^{2 \pi} \int_{0}^{R} E(r, \varphi) d r d \varphi}{\pi R^{2}}
\end{gathered}
$$

is the mean surface irradiance Emean or mean power density.

In this study, we investigated the performances of LED emitters by means of numerical and experimental analysis. The main goal of this study is to obtain a compact and cheap solar simulator for thermal application with class A uniformity. We started to analyze the single LED array, then the three LED arrays configuration (arranged along the vertices of an equilateral triangle) and finally we focused on the four LED arrays setting. The last architecture has been also experimentally investigated, since it is able to provide the demanded uniformity.

In the experimental apparatus, the LED arrays are symmetrically disposed at the corners of a square, which is parallel to the illuminated surface, see fig. 1(c). Each single emitter is realized by an integrated LED array (CXA 3590 from CREE [39]), resulting in a $30 \mathrm{~mm}$ diameter lamp (see figure 1(b)) with a Lambertian light emission distribution represented in fig. 1(a). For this layout the pitch distance (P), i.e. the distance between two adjacent LED arrays' centers, and the LED-to-surface (LS) distance are the two parameters to be considered in order to maximize uniformity on the illuminated surface (in our case $200 \mathrm{~mm} \times 200 \mathrm{~mm}$ ).

\section{Numerical model}

The origin of the coordinate system is at the center of the illuminated surface, which in turn is on the $x-y$ plane $(\mathrm{LS}=0)$, whereas the LEDs are placed on a parallel plane at a positive distance LS along the $\mathrm{z}$ axis, see fig. 1 (c). 
(a)

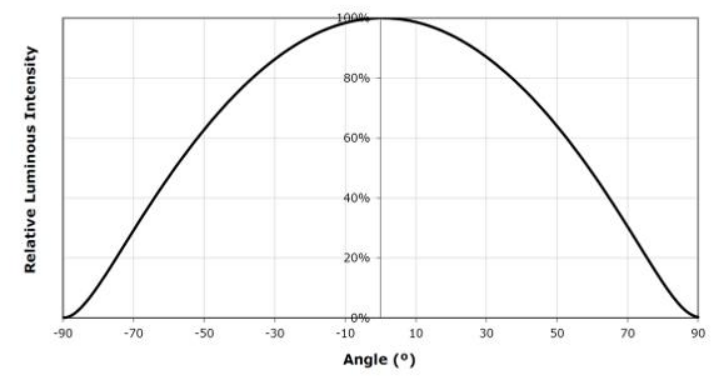

(c)

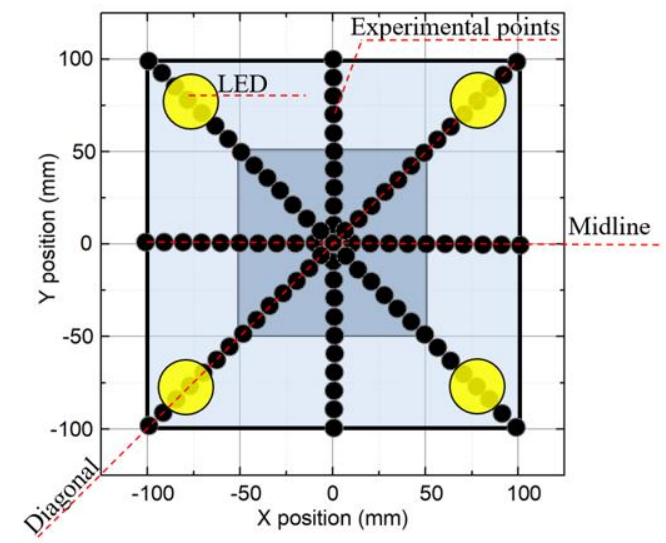

(b)

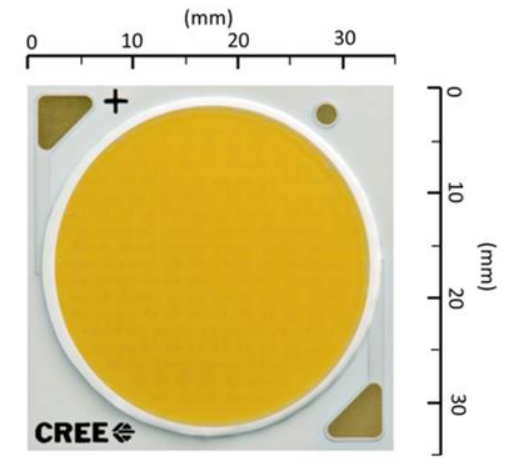

(d)

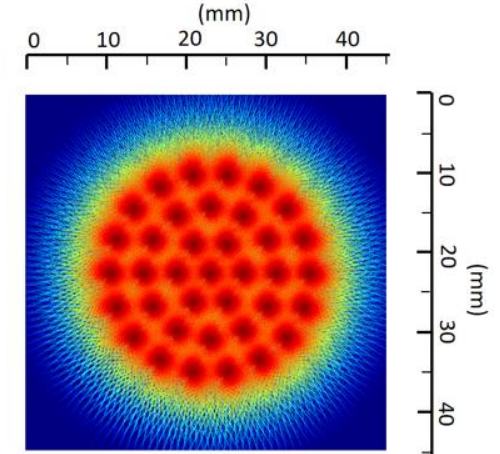

Figure 1. The four-LEDs system features. (a) Angular distribution of the relative luminosity for a single array as reported by the manufacturer; (b) Picture of multi-die integrated LED array forming a single lamp (top view); (c) Spatial arrangement: yellow circles represent LED array positions, blue and light blue squares are the typical and maximum illuminated surface respectively, black dots represent the positions where light power density is measured with a secondary standard pyranometer; (d) colormap of power density light emission of the LED array as reproduced in the ray tracing software by arranging together 37 single Lambertian emitters.

Each LED array is reproduced with 37 punctual Lambertian sources equally spaced in a circle of $30 \mathrm{~mm}$ diameter, as shown in figure 1(d). Every single source numerically emits 30,000 rays (distributed according to the cosine law, eq.1) or 1.5 rays/sq. deg (about 5 rays/msr) on average.

Using a numerical model implemented with the ray tracing module in Comsol Multiphysics, we estimated the device performances in terms of uniformity and mean power density on the illuminated surface. To optimize, we then performed several numerical simulations varying P and LS.

In fig. 2, the simulated irradiance maps and iso-power density lines are reported for three different LED arrays configurations and referred to Class A, Class B and Class C uniformity. Fig. 2(a) refers to a single LED array centered at $(0,0)$ at an LS distance equal to $150 \mathrm{~mm}$, showing the intrinsic non-uniformity of the LED array emission reaching the Class A uniformity only on few $\mathrm{cm}^{2}$. In fig. 2(b) the simulated irradiance of the best configuration for three LED arrays arrangement (distributed at the corners of an equilateral triangle of side $\mathrm{P}$ ) is shown with $\mathrm{LS}=150 \mathrm{~mm}$ and $\mathrm{P}=150 \mathrm{~mm}$. Respect to the single LED array, there is a sensible improvement in terms of achievable power density and size of the high uniformity zone. However, such triangular light pattern provides the required uniformity only on an area of about $16 \mathrm{~cm}^{2}$. Fig. 2(c) reports the irradiance map of the optimal four LED arrays configuration (with a LS $=150 \mathrm{~mm}$ and $\mathrm{P}=160 \mathrm{~mm}$ ). We observe the Class A uniformity in a central zone having an area of about $120 \mathrm{~cm}^{2}$, whereas the Class B is extended on an area of about $250 \mathrm{~cm}^{2}$. Outside such area, the power density rapidly decreases reaching a minimum in correspondence of the blue areas, however Class C is obtained on almost all $400 \mathrm{~cm}^{2}$ investigated. It is worth noting that the four power density peak positions do not match those of the LED arrays (each is slightly shifted towards the center along the diagonals) and that the power density value is higher than in the single array case, due to the superposition effect. With each array emitting $43 \mathrm{~W}$ of light power, the whole four LED configuration provides the typical power density required for solar testing, i.e. $1000 \mathrm{~W} \mathrm{~m}$. Since the results derived from the last configuration satisfy our requirement, additional LED arrays 
175

architectures have not been investigated.

\subsection{Uniformity analysis and optimization}

A series of numerical simulation are carried out varying P and LS parameters to assess the illumination uniformity. The latter is defined in terms of $\Delta E$ parameter, see eq. 3 , on circular areas of radius $R$. The $P$ value is limited to $160 \mathrm{~mm}$ because the whole illumination system is required to have $370 \mathrm{~mm} \times 370 \mathrm{~mm} \times 700 \mathrm{~mm}$ overall dimensions, due to our laboratory constraints. For the same reason, the LS value is limited to $300 \mathrm{~mm}$. Some results obtained in the case of single LED and three LEDs are illustrated in Fig. 3a). Due to the circular symmetry chosen, the results reported in figure 3 are perfectly correlated to figure 2 only in the case of single led, whereas tend to underestimate the area of uniformity.
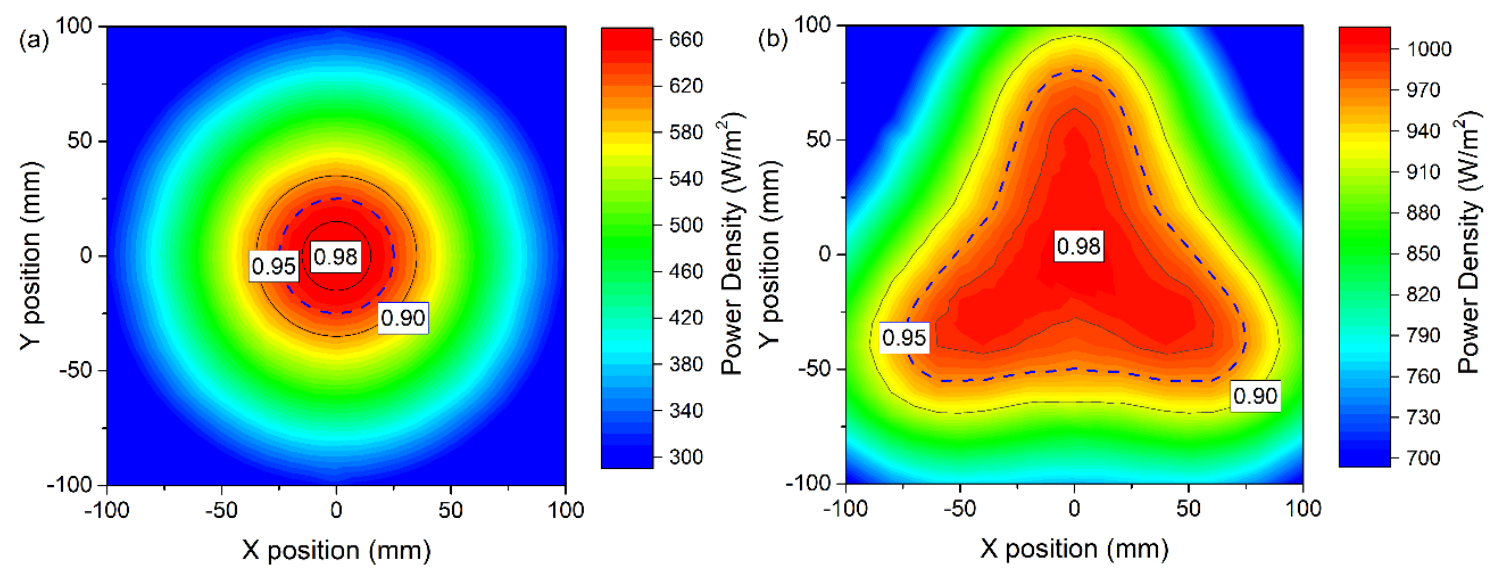

(c)

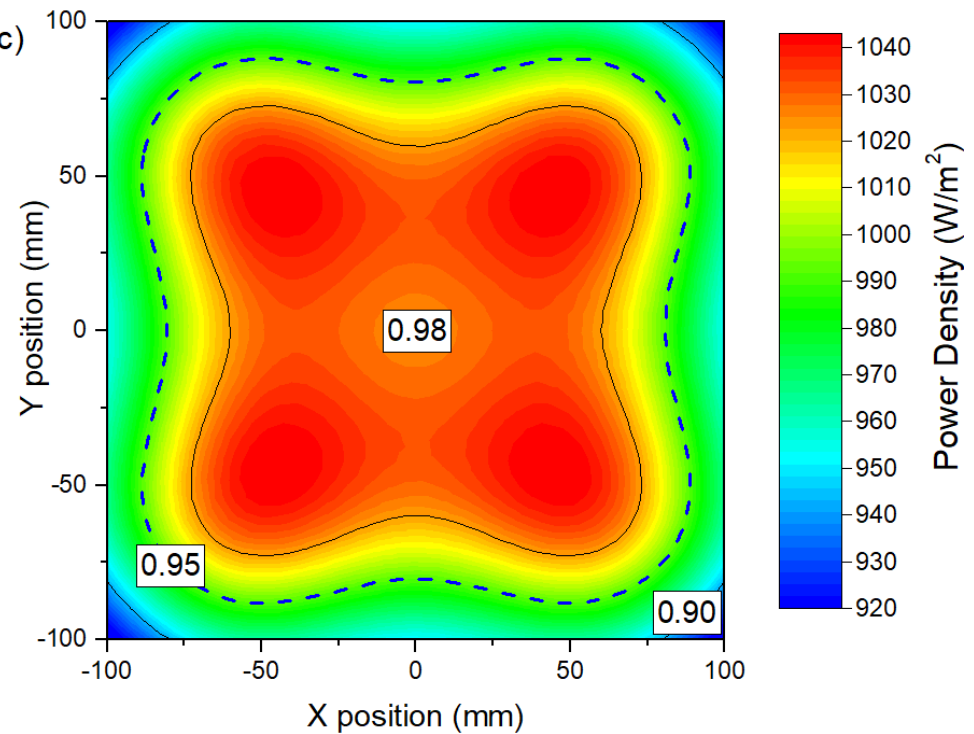

Figure 2. The simulated power density colormaps and iso-power density lines (Black for 0.98 and 0.9 , dashed blue for 0.95) on the illuminated $200 \mathrm{~mm} \times 200 \mathrm{~mm}$ surface with each LED array emitting 43 $\mathrm{W}$ of light power. (a) Single LED array placed at $(0,0) \mathrm{mm}, \mathrm{LS}=150 \mathrm{~mm}$ : power density is maximum at the array center and has a steep decrease; (b) Three LED arrays with $P=160 \mathrm{~mm}$ and $\mathrm{LS}=150 \mathrm{~mm}$ : such architecture improves power density and uniformity without satisfy our requirements; (c) Four LED arrays with $\mathrm{P}=160 \mathrm{~mm}$ and $\mathrm{LS}=150 \mathrm{~mm}$ : the maxima are slightly displaced in respect to the arrays center by the superposition effect and uniformity dramatically increases. The line of $90 \%$ uniformity is present only in the four corners of the color map.

Figure $3 b$ ) shows the results obtained in the four LED arrays configuration. The maximum uniformity is found for $\mathrm{P}=160 \mathrm{~mm}$ and $\mathrm{LS}=150 \mathrm{~mm}$, with a $\triangle \mathrm{E}$ of just $2 \%$ and $5 \%$ on an illuminated area of $50 \mathrm{~mm}$ and $75 \mathrm{~mm}$ radius respectively. Varying LS in between 140 and $160 \mathrm{~mm}$, 
198

199

still provides high uniformity with $\Delta E$ respectively below $3 \%$ and $5 \%$. A $20 \mathrm{~mm}$ smaller pitch distance $(\mathrm{P}=140 \mathrm{~mm})$ is required to observe a variation, at least for larger radii.

(a)

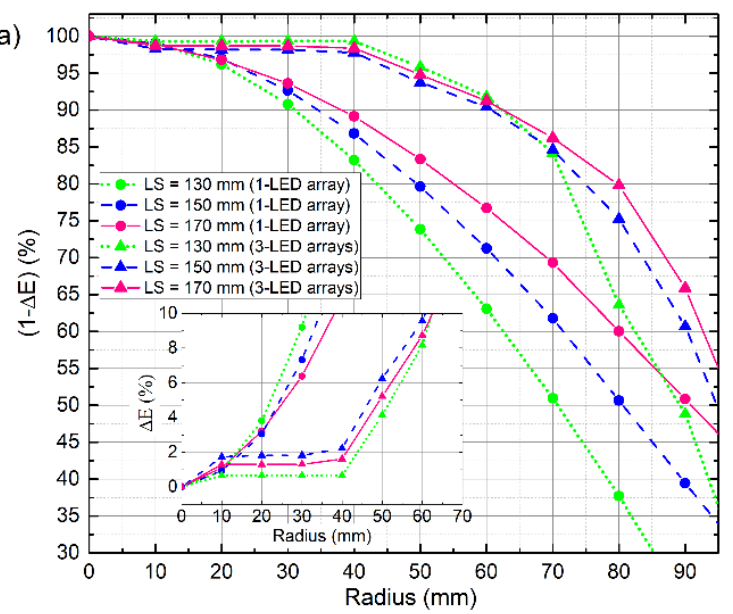

(b)

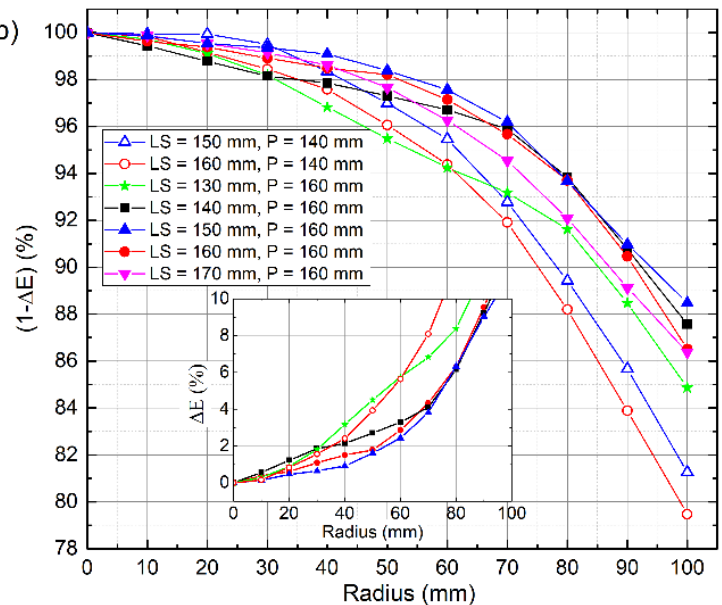

Figure 3. Variation of non-uniformity parameter (and its complement in the inset) for different $P$ and LS values as a function of the radius of the illuminated surface. a) Single LED array and equilateral triangle configuration. b) Four LED arrays on corners of a square. In both figures the inset shows details of the high uniformity zone.

Such results indicate that a slight variation of P or LS from the previous optimal values of $160 \mathrm{~mm}$ and $150 \mathrm{~mm}$, respectively, does not severely affect uniformity, particularly when samples smaller than 75 $\mathrm{mm}$ radius are illuminated. This greatly simplify the experimental set-up fabrication where millimetric assembly accuracy is enough to maintain the required performances.

\subsection{Power density analysis and optimization}

The mean power density is also computed as function of the illuminated area radius for different $L S$ values $(P=160 \mathrm{~mm})$. It varies from $26 \%$ to $20 \%$ of the total emitted power, for $\mathrm{LS}$ of 130 $\mathrm{mm}$ and $170 \mathrm{~mm}$ respectively. This reduction effect can be roughly estimated at $0.3 \%$ per millimeter $(100 \mathrm{~W}$ for $30 \mathrm{~mm})$, as shown in fig. 4(a). Proper knowledge of power density derivative along the radial direction can also be useful to quantify errors related to sample positioning. For all the geometrical configurations previously analyzed and for a sample mis-positioning of few millimeters, we found a variation that would reflect in a total mean power density error below $1 \%$, see fig. 4 (b).
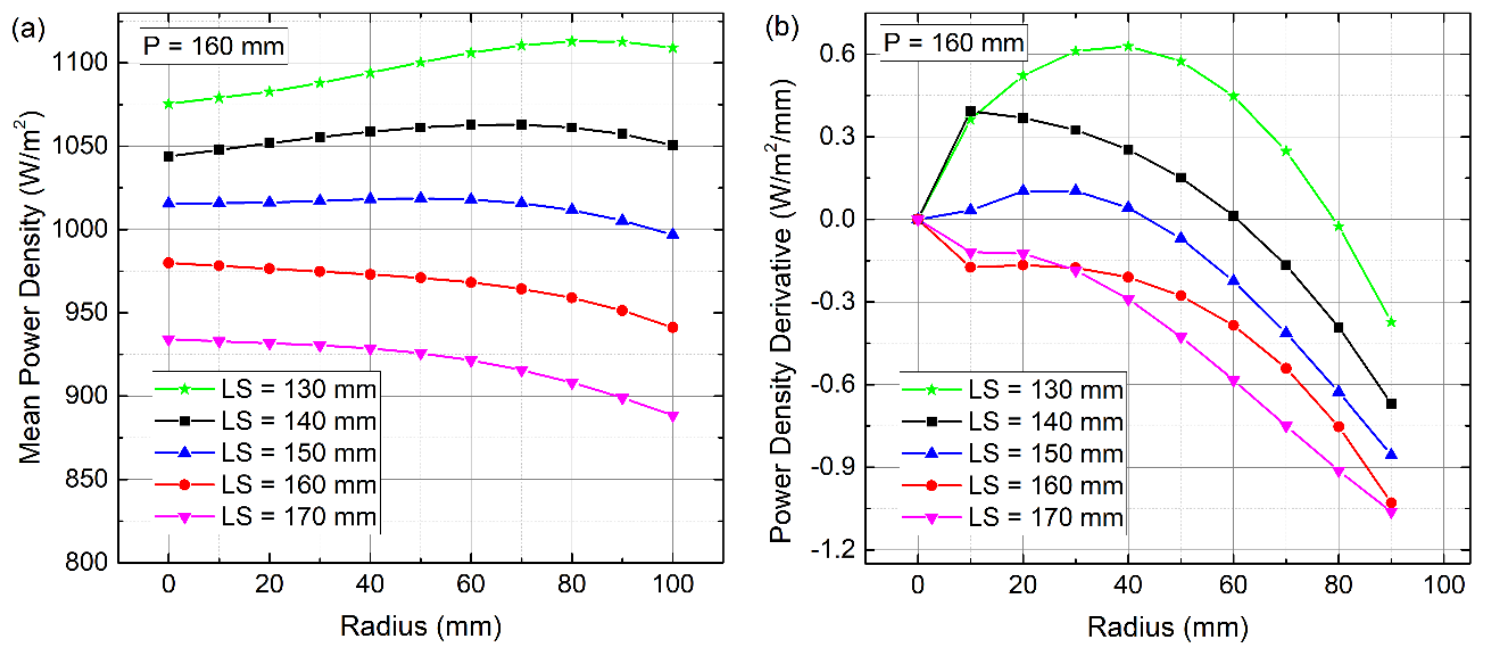

Figure 4. a) The mean value of irradiance, $E_{m e a n}$, on the illuminated surface for different LS values $(P=160 \mathrm{~mm})$. The light power emitted by each LED array is $43 \mathrm{~W}$. b) Mean power density derivative along radial direction as a function of the radius of the illuminated surface. 
It is worth mentioning that the amount of light power that reaches the illuminated surface is only a fraction of the total emitted by the four LED arrays. Such light loss, mainly spilling from the sides, might make the workroom less comfortable and should be reduced. For this reason, the experimental apparatus has been equipped with black screens to optically isolate the illumination chamber. These screens have been experimentally characterized by means of reflectivity measurements performed with Integrating Sphere coupled to an Optical Spectrum Analyzer. Results report high absorbance in the visible spectrum, more than $96 \%$. Ray tracing simulations confirm that the amount of the light reflected by the black screens and/or by the supporting structure and then projected towards the $200 \mathrm{~mm} \times 200$ $\mathrm{mm}$ target surface is much less than $1 \%$ in terms of power density. The reflected light is mainly directed to the frame area, i.e. the area that fill the gap between the $200 \mathrm{~mm} \times 200 \mathrm{~mm}$ targeted surface and 260 $\mathrm{mm} \times 260 \mathrm{~mm}$ area confined by the black screens.

\section{Experimental apparatus and results}

In fig. 5(a) the adopted LED lamp structure is reported: it consists of four LED arrays mounted on six rectangular stainless steel bars assembled using bolts and nuts; slots in the bars allow to adjust the pitch distance P; the whole lamp has dimension of $26 \mathrm{~cm} \times 26 \mathrm{~cm} \times 8 \mathrm{~cm}$. The LED lamp is mounted in a supporting structure containing all the control electronics as shown in the picture reported in Fig. 5 (b). The guides in the support pillars allow to accommodate samples with different heights and to easily adjust the LS distance according to measurement requirements. The whole system (including the control electronics) fits in a volume of $70 \mathrm{~cm} \times 37 \mathrm{~cm} \times 37 \mathrm{~cm}$, therefore representing a suitable workbench solution for laboratory activities.

The four white high power LED arrays CREE CXA3590 were from the same fabrication lot assuring a $1 \%$ uniformity of relevant parameters [39]. Each LED array is powered by its own LEDdriver ELG-200-C1750 from Mean Well [40] and the light output can be controlled by an external 0-10 V DC voltage acting as dimmer. The whole LED system is controlled by a combination of a custom PCB board, a National Instruments (NI) USB-6008/6009 OEM device [41] and a LabView software. The dimmers of the four LED drivers are controlled by analog outputs of the USB NI board. Because the NI board is equipped with just two analog outputs, one output controls two LED drivers. Also, these outputs range from 0 to $+5 \mathrm{~V}$, while the dimmer requires $0 \mathrm{~V}$ to $+10 \mathrm{~V}$ for dimming and therefore voltage doubler circuits are provided on the custom PCB board. The four voltage doubler circuits can be fine regulated by means of trimmers. This feature is used to equalize light output by placing a pyranometer directly under the four individual LED arrays.

(a)

(b)

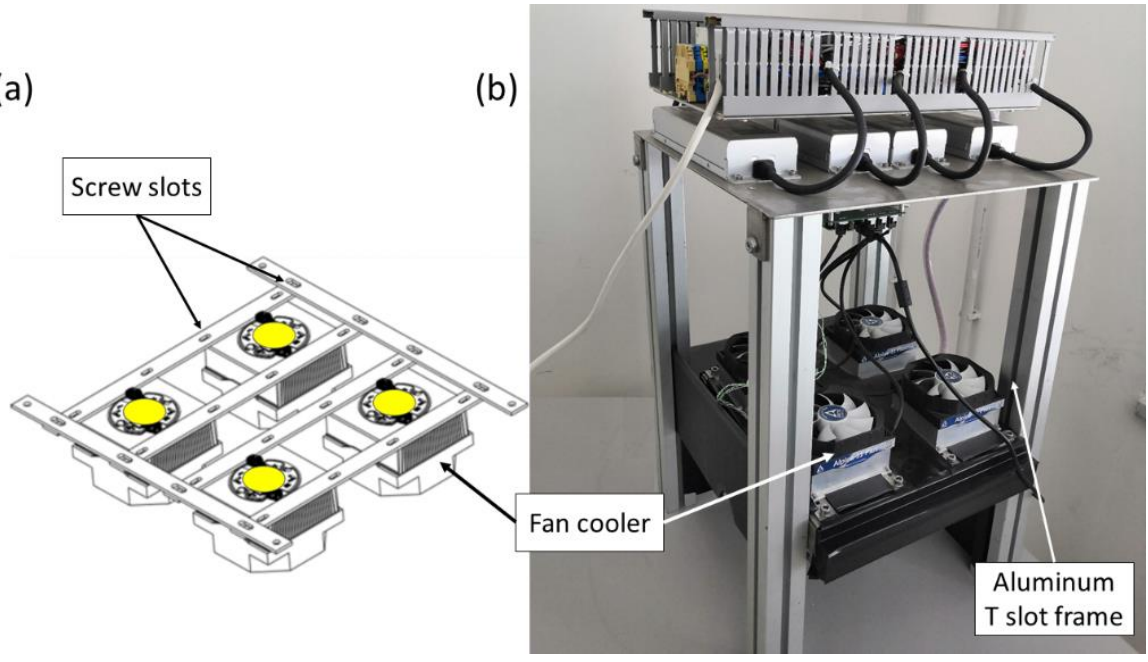

Figure 5. a) The LED lamp is composed of four identical LED arrays, symmetrically arranged with a pitch distance of $160 \mathrm{~mm}$, which can be modified by screw slots. b) The supporting structure is designed to accommodate the electronic control and to make the LS distance tunable via the T-Slot in 
LED arrays are turned on and off using two digital outputs of the NI board, driving solid state relays of appropriate power rating. Again, one digital output controls two LED drivers simultaneously. LED array temperatures are monitored with a K-type thermocouple attached to the chip socket and readings performed using a precision thermocouple amplifier with cold junction compensation [42], connected to an analog input channel of the NI Board.

The luminous flux emitted by the LED arrays strongly depends on the LED temperature [39]. We therefore mounted each LED array chip on a modified CPU cooler of double power rating [43]. Such solution allows to keep the LED temperature stable during operation at values below $75{ }^{\circ} \mathrm{C}$ for any applied bias voltages.

In order to optically seal the illuminating chamber, black shields are disposed around the illuminated surface, fig. 5(b). This solution provides a dual advantage: first, the illuminated surface is shielded against any external light source; second, the light emitted by the system is confined within, keeping the workroom more comfortable.
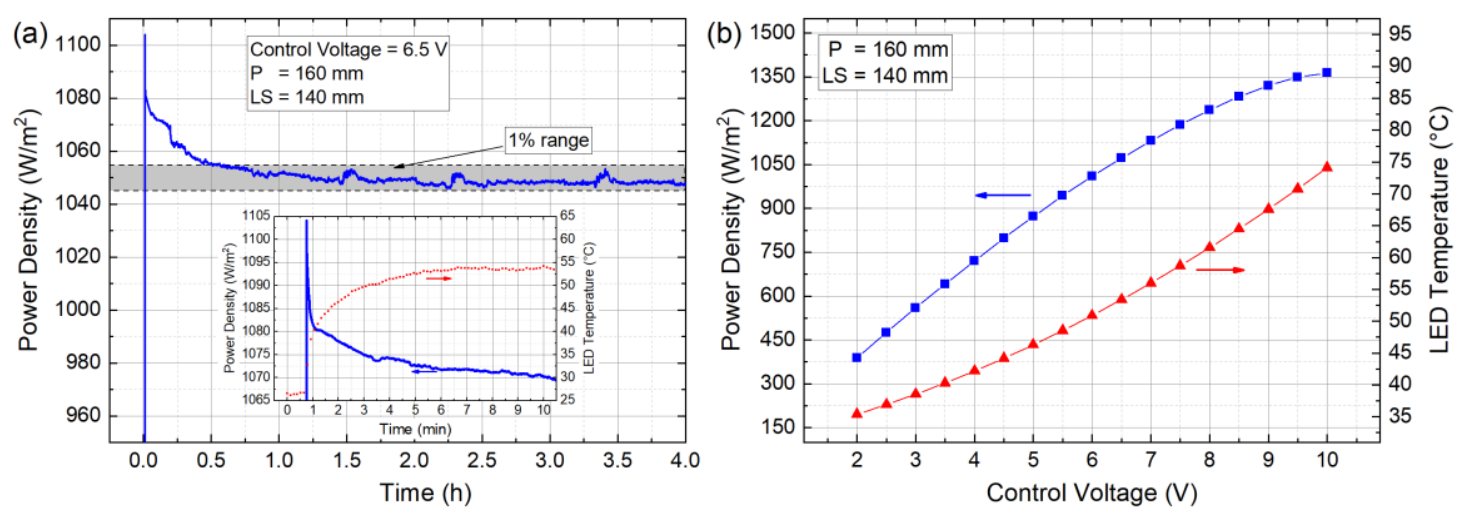

Figure 6. LED array light output stability. (a) Evolution of light power density with time: the gray box indicates $1 \%$ range of uncertainty around the steady state level. The inset shows the inverse correlation between emitted power and LED operating temperature. (b) Steady state power density and operating temperature as a function of control voltage (error bars are within the size of the data marker).

The experimental characterization of the LED array assembly has been conducted by using a secondary standard pyranometer (CMP11 from Kipp \& Zonen [44]) with 1\% precision and a response time of less than $5 \mathrm{~s}$. Firstly, a time-stability test has been conducted, see fig. 6(a): the pyranometer is placed directly under the LED array at a distance of $140 \mathrm{~mm}$ with a control voltage of $6.5 \mathrm{~V}$ and the power density is read out together with the LED array temperature. After $30 \mathrm{~min}$ of illumination, the measured power density becomes stable in time within the uncertainty of the pyranometer. In particular, the inset of fig. 6(a) reveals that the reduction of power output with time can be directly linked to the thermal stabilization of the LED source. The behavior reported in figure fig. 6(a) is identical for the four LEDs and the stabilized power density provided by each LED array is identical within 1\% make the LED lamp a class A in term of temporal instability Secondly, a series of linearity measurements, varying the control voltage from $2 \mathrm{~V}$ to $10 \mathrm{~V}$, were carried out. Also in this case, stable power output is reached $30 \mathrm{~min}$ after switching on the LED arrays, while power density and temperature values depend from the control voltage as reported in fig. 6(b). Power density level can be varied from $390 \mathrm{~W} \mathrm{~m}^{-2}$ up to $1360 \mathrm{~W} \mathrm{~m}^{-2}$, with almost perfect linearity, making the device suitable for both low and high intensity applications.

An illumination map has been measured for a fixed pitch of $160 \mathrm{~mm}$ for several LS distances and control voltages. The black dots in fig. 1(c) represent the pyranometer measurement positions: 96 points radially disposed on the maximum $200 \mathrm{~mm}$ x $200 \mathrm{~mm}$ illuminated surface with a step of 10 $\mathrm{mm}$. Radial mapping is preferred, since power density peaks and valleys are expected to be along diagonals and symmetry lines, see Fig. 1(c) and Fig. 2(b). The experimental results obtained for a control voltage of $6.5 \mathrm{~V}$ and a LS distance of 130, 140 and $150 \mathrm{~mm}$ are summarized in fig. 7(a) and compared to the corresponding numerical simulation. Each experimental point is obtained by 
298

299

300

301

302

303

304

305

306

307

308

averaging the eight experimental data acquired at the same radial distance from the center (the error bars represent $1 \%$ uncertainty from the pyranometer calibration). A very good accordance is found, proving that the numerical model is reliable and properly validated. Similar results are found also when analyzing the power density along the midlines and the diagonals as shown in Fig. 7(b) for $\mathrm{P}=160 \mathrm{~mm}$ and $\mathrm{LS}=150 \mathrm{~mm}$. A maximum deviation of $1 \%$ is also found for other voltages and LS distances confirming the quality of our experimental set-up.
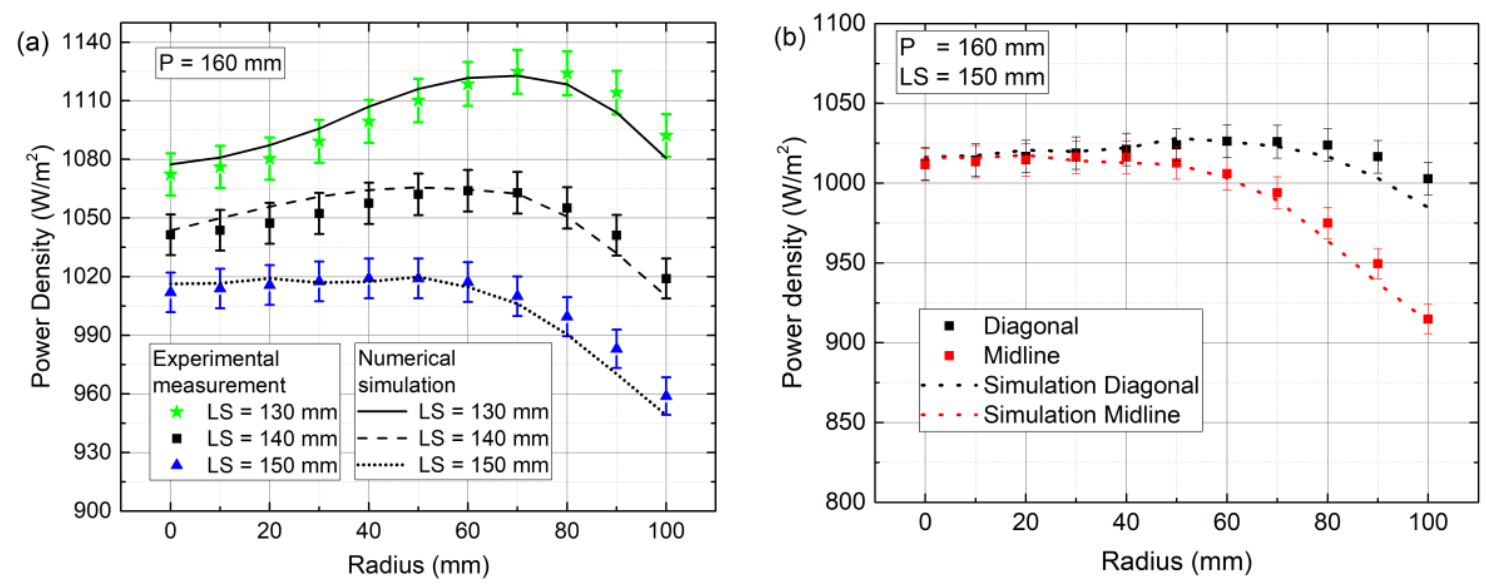

Figure 7. a) Power density average on 8 points measured at each reported radius (dots) and simulation result in the same points (lines). b) Comparison between simulated and experimental results along symmetry and diagonal lines. In a) and b) the measured point error bars refer to $1 \%$ pyranometer uncertainty.

\section{Conclusion}

Several research fields and numerous laboratory activities require an artificial light source of high intensity and high uniformity. Low cost commercial devices offer uniformity of the order of $95 \%$ or less, while higher uniformity is reached only with more expensive solution (including mirrors and lenses). In this paper we have presented a compact and low-cost LED lamp providing higher than $95 \%$ uniformity when illuminating an area up to $200 \mathrm{~cm}^{2}$ with a power density in excess of $1000 \mathrm{Wm}^{-}$ 2. It consists of an actively cooled square arrangement of four high power white LED arrays illuminating a maximum of surface $200 \mathrm{~mm} \times 200 \mathrm{~mm}$ and having a volume of $260 \mathrm{~mm} \times 260 \mathrm{~mm} \times$ $80 \mathrm{~mm}$. The control electronic has been custom developed for individual fine-tuning of each LED array light output. A LabVIEW program allows controlling and monitoring the system. The LED lamp has been mounted in a supporting structure to experimentally validate the ray tracing simulations. Suitable guides in the support pillars allow to easily adjust the distance between the light source and the illuminated surface to satisfy different measurement requirements. The system has been used to characterize the efficiency of selective solar absorber [45].

Author Contributions: Conceptualization, Francesco Di Giamberardino, Vittorio Palmieri and Roberto Russo; Data curation, Carmine D' Alessandro, Davide De Maio and Daniela De Luca; Formal analysis, Carmine D' Alessandro; Investigation, Teresa Mundo, Matteo Monti and Davide Dalena; Methodology, Carmine D' Alessandro, Davide De Maio, Marilena Musto and Roberto Russo; Project administration, Vittorio Palmieri; Resources, Vittorio Palmieri; Software, Carmine D' Alessandro, Davide De Maio and Francesco Di Giamberardino; Supervision, Marilena Musto and Roberto Russo; Visualization, Daniela De Luca and Emiliano Di Gennaro; Writing - original draft, Carmine D' Alessandro; Writing - review \& editing, Marilena Musto, Emiliano Di Gennaro and Roberto Russo.

Acknowledgment: The authors are grateful to Rosario Iameo for his help in programming the data acquisition software. The PhD grant of one of the authors (DDL) is funded by the PON 20142020 "Dottorati innovativi con caratterizzazione industriale, XXXIV ciclo" program. The PHD grant of one of the author (DDM) is financed by the CNR-Confindustria agreement for industrial PhD 
grants.

\section{References}

1. Kruisselbrink, T.; Dangol, R.; Rosemann, A. Photometric measurements of lighting quality: An overview. Building and Environment 2018, 138, 42-52, doi:10.1016/j.buildenv.2018.04.028.

2. Gago-Calderón, A.; Hermoso-Orzáez, M.; De Andres-Diaz, J.; Redrado-Salvatierra, G. Evaluation of Uniformity and Glare Improvement with Low Energy Efficiency Losses in Street Lighting LED Luminaires Using Laser-Sintered Polyamide-Based Diffuse Covers. Energies 2018, 11, 816, doi:10.3390/en11040816.

3. Knowles, J.P.; Elliott, L.D.; Booker-Milburn, K.I. Flow photochemistry: Old light through new windows. Beilstein J. Org. Chem. 2012, 8, 2025-2052, doi:10.3762/bjoc.8.229.

4. Maurya, R.A.; Park, C.P.; Kim, D.-P. Triple-channel microreactor for biphasic gas-liquid reactions: Photosensitized oxygenations. Beilstein J. Org. Chem. 2011, 7, 1158-1163, doi:10.3762/bjoc.7.134.

5. Roibu, A.; Morthala, R.B.; Leblebici, M.E.; Koziej, D.; Van Gerven, T.; Kuhn, S. Design and characterization of visible-light LED sources for microstructured photoreactors. React. Chem. Eng. 2018, 3, 849-865, doi:10.1039/C8RE00165K.

6. Haas, C.P.; Roider, T.; Hoffmann, R.W.; Tallarek, U. Light as a reaction parameter - systematic wavelength screening in photochemical synthesis. React. Chem. Eng. 2019, 4, 1912-1916, doi:10.1039/C9RE00339H.

7. Kheyrandish, A.; Taghipour, F.; Mohseni, M. UV-LED radiation modeling and its applications in UV dose determination for water treatment. Journal of Photochemistry and Photobiology A: Chemistry 2018, 352, 113121, doi:10.1016/j.jphotochem.2017.10.047.

8. Ogonowska, P.; Woźniak, A.; Pierański, M.; Wasylew, T.; Kwiek, P.; Brasel, M.; Grinholc, M.; Nakonieczna, J. Application and characterization of light-emitting diodes for photodynamic inactivation of bacteria. Lighting Research \& Technology 2019, 51, 612-624, doi:10.1177/1477153518781478.

9. Sikora, A.; Janus, P.; Sierakowski, A. The impact of the light exposure on the morphological properties of selected photoresists. OPTICA APPLICATA; 01/2019; ISSN 1429-7507 2019, doi:10.5277/OA190116.

10. Shiba, S.F.; Tan, J.Y.; Kim, J. Multidirectional UV-LED lithography using an array of high-intensity UVLEDs and tilt-rotational sample holder for 3-D microfabrication. Micro and Nano Syst Lett 2020, 8, 5, doi:10.1186/s40486-020-00107-y.

11. Dubovikov, A.L.; Repin, S.S.; Natarovskii, S.N. Features of the use of LEDs in artificial-vision systems. J. Opt. Technol. 2005, 72, 40, doi:10.1364/JOT.72.000040.

12. Wu, X.; Gao, G. LED light design method for high contrast and uniform illumination imaging in machine vision. Appl. Opt. 2018, 57, 1694, doi:10.1364/AO.57.001694.

13. Wang, X. LED ring array light source design and uniform illumination properties analysis. Optik 2017, 140, 273-281, doi:10.1016/j.ijleo.2017.04.045.

14. Pan, C.-T.; Francisco, M.D.; Yen, C.-K.; Wang, S.-Y.; Shiue, Y.-L. Vein Pattern Locating Technology for Cannulation: A Review of the Low-Cost Vein Finder Prototypes Utilizing near Infrared (NIR) Light to Improve Peripheral Subcutaneous Vein Selection for Phlebotomy. Sensors 2019, 19, 3573, doi:10.3390/s19163573.

15. Dong, J.; Xiong, D. Applications of Light Emitting Diodes in Health Care. Ann Biomed Eng 2017, 45, 25092523, doi:10.1007/s10439-017-1930-5.

16. Moss, R.W.; Shire, G.S.F.; Eames, P.C.; Henshall, P.; Hyde, T.; Arya, F. Design and commissioning of a virtual image solar simulator for testing thermal collectors. Solar Energy 2018, 159, 234-242, doi:10.1016/j.solener.2017.10.044.

17. Grandi, G.; Ienina, A.; Bardhi, M. Effective Low-Cost Hybrid LED-Halogen Solar Simulator. IEEE Trans. on Ind. Applicat. 2014, 50, 3055-3064, doi:10.1109/TIA.2014.2330003.

18. Hirsch A New $75 \mathrm{~kW}$ High-Flux Solar Simulator for High-Temperature Thermal and Thermochemical Research. Journal of Solar Energy Engineering 15.

19. Meng, Q.; Wang, Y.; Zhang, L. Irradiance characteristics and optimization design of a large-scale solar simulator. Solar Energy 2011, 85, 1758-1767, doi:10.1016/j.solener.2011.04.014.

20. Esen, V.; Sağlam, S..; Oral, B. Solar Irradiation Fundamentals and Solar Simulators. In A Practical Guide for Advanced Methods in Solar Photovoltaic Systems; Mellit, A., Benghanem, M., Eds.; Springer International Publishing: Cham, 2020; pp. 3-28 ISBN 978-3-030-43473-1.

21. Dong, X.; Sun, Z.; Nathan, G.J.; Ashman, P.J.; Gu, D. Time-resolved spectra of solar simulators employing metal halide and xenon arc lamps. Solar Energy 2015, 115, 613-620, doi:10.1016/j.solener.2015.03.017.

22. Dibowski, G.; Esser, K. Hazards Caused by UV Rays of Xenon Light Based High Performance Solar 
Simulators. Safety and Health at Work 2017, 8, 237-245, doi:10.1016/j.shaw.2016.12.002.

23. Bazzi, A.M.; Klein, Z.; Sweeney, M.; Kroeger, K.P.; Shenoy, P.S.; Krein, P.T. Solid-State Solar Simulator. IEEE Trans. on Ind. Applicat. 2012, 48, 1195-1202, doi:10.1109/TIA.2012.2199071.

24. Lopez-Fraguas, E.; Sanchez-Pena, J.M.; Vergaz, R. A Low-Cost LED-Based Solar Simulator. IEEE Trans. Instrum. Meas. 2019, 68, 4913-4923, doi:10.1109/TIM.2019.2899513.

25. Al-Ahmad, A.Y.; Holdsworth, J.; Vaughan, B.; Sharafutdinova, G.; Zhou, X.; Belcher, W.J.; Dastoor, P.C. Modular LED arrays for large area solar simulation. Prog Photovolt Res Appl 2019, 27, 179-189, doi:10.1002/pip.3072.

26. Tawfik, M.; Tonnellier, X.; Sansom, C. Light source selection for a solar simulator for thermal applications: A review. Renewable and Sustainable Energy Reviews 2018, 90, 802-813, doi:10.1016/j.rser.2018.03.059.

27. Yang, H.; Bergmans, J.W.M.; Schenk, T.C.W.; Linnartz, J.-P.M.G.; Rietman, R. An analytical model for the illuminance distribution of a power LED. Opt. Express 2008, 16, 21641, doi:10.1364/OE.16.021641.

28. Wang, K.; Wu, D.; Qin, Z.; Chen, F.; Luo, X.; Liu, S. New reversing design method for LED uniform illumination. Opt. Express 2011, 19, A830, doi:10.1364/OE.19.00A830.

29. Su, Z.; Xue, D.; Ji, Z. Designing LED array for uniform illumination distribution by simulated annealing algorithm. Opt. Express 2012, 20, A843, doi:10.1364/OE.20.00A843.

30. Yu, X.; Wei, X.; Zhang, O.; Zhang, X. Research on illumination uniformity of high-power LED array light source.; Hangzhou, China, 2018; p. 050019.

31. Moreno, I.; Avendaño-Alejo, M.; Tzonchev, R.I. Designing light-emitting diode arrays for uniform nearfield irradiance. Appl. Opt. 2006, 45, 2265, doi:10.1364/AO.45.002265.

32. Whang, A.J.-W.; Yi-Yung Chen; Yuan-Ting Teng Designing Uniform Illumination Systems by SurfaceTailored Lens and Configurations of LED Arrays. J. Display Technol. 2009, 5, 94-103, doi:10.1109/JDT.2008.2001865.

33. Kennedy, C.E. Review of Mid- to High-Temperature Solar Selective Absorber Materials; 2002; p. NREL/TP-52031267, 15000706;

34. Kraemer, D.; McEnaney, K.; Cao, F.; Ren, Z.; Chen, G. Accurate determination of the total hemispherical emittance and solar absorptance of opaque surfaces at elevated temperatures. Solar Energy Materials and Solar Cells 2015, 132, 640-649, doi:10.1016/j.solmat.2014.10.026.

35. Granados, L.; Takamure, N.; Bing, J.; Huang, S.; Merhvarz, H.; McKenzie, D.R.; Ho-Baillie, A. Direct Determination of Total Hemispherical Emittance of Perovskite and Silicon Solar Cells. Cell Reports Physical Science 2020, 1, 100008, doi:https://doi.org/10.1016/j.xcrp.2019.100008.

36. Xu, K.; Du, M.; Hao, L.; Mi, J.; Yu, Q.; Li, S. A review of high-temperature selective absorbing coatings for solar thermal applications. Journal of Materiomics 2020, 6, 167-182, doi:10.1016/j.jmat.2019.12.012.

37. Russo, R.; Monti, M.; di Giamberardino, F.; Palmieri, V.G. Characterization of selective solar absorber under high vacuum. Opt. Express 2018, 26, A480, doi:10.1364/OE.26.00A480.

38. Wood, D. Optoelectronic semiconductor devices; Prentice-Hall international series in optoelectronics, Prentice Hall PTR, 1994; 3rd ed.; McGraw-Hill: Boston, 2003; ISBN 978-0-07-232107-4.

39. CREE. CreeQR XLampQR CXA3590 LED LED Description, 2019.

40. Mean Well. ELG-200-C-S1750B from Mean Well, https://www.meanwellweb.com/content/files/pdfs/productPdfs/MW/ELG-200-C/ELG-200-C-spec.pdf.

41. National Instruments Corporation. NI USB-6008/6009 OEM, www.ni.com/pd f/manuals/371728b.pdf.

42. ANALOG DEVICES. Precision Thermocouple Amplifiers with Cold Junction Compensation, www.analog.com/media/en/technical - documentation/data - sheets/ad8494/8495/8496/8497.pdf.

43. ARCTIC. Alpine 11 PLUS Cooler Description, 2011.

44. Kipp \& Zonen, CMP11, https://www.kippzonen.com/Download/73/Instruction-Sheet-Pyranometers-CMP-series.

45. Russo, R.; et al. Characterization of selective solar absorbers under Sun and LED illumination in high vacuum in preparation. 